\title{
Espirometría: Manual de procedimientos. Sociedad Chilena de Enfermedades Respiratorias, 2006*
}

\author{
MÓNICA GUTIÉRREZ C. (coordinadora), TERESA BEROÍZA W., GISELLA BORZONE T., \\ IVÁN CAVIEDES S., JUAN CÉSPEDES G., MÓNICA GUTIÉRREZ N., RODRIGO MORENO B., \\ MANUEL OYARZÚN G., SYLVIA PALACIOS M. y PATRICIA SCHONFFELDT G.
}

\section{Manual of Procedures on Spirometry. Chilean Society of Respiratory Diseases, 2006}

Spirometry is a essential test for assessing pulmonary function. This test is frequently used in clinical practice and population studies. Among the several indices derived from forced expiration, forced expiratory volume in the $1^{\text {st }}$ second $\left(F E V_{1}\right)$ and forced vital capacity $(F V C)$ ) are the most commonly used because of good reproducibility, ease of measurement, and correlation with disease stage, functional condition, morbidity and mortality. Considering the importance to apply standardized techniques for measuring spirometric indices, a committee of specialists on pulmonary function from the Chilean Society of Respiratory Diseases, has elaborated this manual of procedures on spirometry. The aim of this manual is to report the international standards for carrying out spirometry, to promote their application in clinical and laboratory practice. Besides a general assent way for interpreting and reporting spirometric values at clinical and at laboratory work is proposed.

Key words: Spirometry; standardized procedures; interpretation; acceptability criteria.

\section{Resumen}

La espirometría es una prueba fundamental en la evaluación funcional respiratoria. Este test es utilizado frecuentemente en la práctica clínica y en estudios de poblaciones. Entre los diversos índices derivados de una espiración forzada, el VEF 1 y la CVF son los más usados debido a su buena reproducibilidad, facilidad de su medición, y su grado de correlación con la etapa de la enfermedad, condición funcional, morbilidad y mortalidad. Considerando la importancia de aplicar procedimientos estandarizados para realizar espirometrías, un comité de especialistas en función pulmonar de la Sociedad Chilena de Enfermedades Respiratorias, elaboró este manual de procedimientos para realizar espirometrías. El objetivo de este manual es comunicar las normas internacionales para realizar espirometrías, a fin de promover su aplicación en la práctica clínica y de laboratorio. Además se propone una manera consensuada para informar e interpretar los valores espirométricos tanto en la práctica clínica como en el trabajo de laboratorio.

Palabras clave: Espirometría; procedimientos estandarizados; interpretación; criterios de aceptabilidad.

Índice

I. Consideraciones generales: Espacio físico. Personal. Calificación técnica.

II. Equipos: Tipos. Requerimientos mínimos.

III. Controles de calidad de los equipos.

IV. Indicaciones.

V. Contraindicaciones.

VI. Recomendaciones al paciente previas al examen.

VII. Técnica: Higiene y control de infecciones.
Preparación del paciente. Ejecución del examen y procedimientos.

Respuesta a broncodilatador.

VIII. Criterios de aceptabilidad y reproducibilidad

IX. Registro de resultados.

X. Interpretación del informe espirométrico en el laboratorio.

XI. Informe final.

XII. Interpretación clínica del informe espirométrico.

XIII.Bibliografía.

* Sección Función Pulmonar Adultos, Sociedad Chilena de Enfermedades Respiratorias. 25 de noviembre, 2006. 
La espirometría es un examen fundamental en la evaluación de la función pulmonar. De los diversos índices derivados de una espiración forzada, el $\mathrm{VEF}_{1}$ y la CVF son los más usados debido a su buena reproducibilidad, facilidad de su medición, y grado de correlación con la etapa de la enfermedad, condición funcional, morbilidad y mortalidad. Este manual pretende entregar la normativa vigente a nivel internacional sobre indicaciones, técnica, criterios de aceptabilidad y reproducibilidad e información mínima que debe incluir el informe final.

\section{Consideraciones Generales}

\section{a) Espacio físico}

Idealmente debe consistir en una habitación de al menos 2 × 3 m, dedicada exclusivamente a esta técnica. Debe poder acomodar al paciente, al espirómetro y a quien realiza la espirometría. Debe contar con una forma de medir la talla o estatura, una pesa, un termómetro ambiental, un barómetro de mercurio, un sillón cómodo (de brazos) para el paciente y un mueble o estante para colocar pinzas nasales y otros elementos o accesorios del equipo.

\section{b) Personal}

Si se trata de un laboratorio de función pulmonar, un médico especialista en enfermedades respiratorias debe estar a cargo del informe, interpretación y supervisión en la realización de la técnica, la cual deberá ser ejecutada por profesionales de la salud (idealmente tecnólogo-médico). Todos ellos deben estar capacitados y certificados en la técnica.

Cuando la espirometría se realiza fuera de un laboratorio especializado, el operador debe estar calificado y certificado por un centro especializado que a su vez esté certificado como idóneo para capacitar a terceros. En este caso también deberá estar bajo la supervisión del médico a cargo del laboratorio de función pulmonar del área correspondiente.

En todo caso cualquiera sea el lugar donde se realice la espirometría, el responsable del cumplimiento de las normas y del informe del examen, será el médico designado para tales funciones.

\section{c) Calificación del técnico en espirometrías}

La espirometría será realizada por médico con capacitación en función pulmonar y tecnólogo médico o técnico paramédico con vasta experiencia en este examen. Para la realización de espirometrías se requiere una formación de un mes de trabajo supervisado, instrucción en los fundamentos biológicos, conceptos de fisiología y fisiopatología respiratoria y nociones de patología respiratoria relacionada. El aspirante a técnico debe mostrar capacidad de relación con pacientes y para el trabajo en equipo. La experiencia que permite la detección de errores en el procedimiento y en el funcionamiento de los aparatos, que capacita al técnico para trabajar con cierta autonomía, suele alcanzarse luego de seis meses de entrenamiento.

\section{Equipos}

Actualmente, existen dos tipos de espirómetros: de volumen y de flujo.

Los espirómetros de flujo (neumotacómetros) son los más utilizados. Miden el flujo a partir de una resistencia conocida que produce una diferencia de presión entre uno y otro lado del paso del aire (neumotacómetro tipo Fleish). También se han comercializado espirómetros que utilizan otro tipo de neumotacómetros para medir flujo: turbina, pistón, sensor de temperatura, ultrasonido, etc.

Los equipos que poseen neumotacómetros asociados a un sistema computarizado, pueden ser divididos en dos grupos:

a) Equipos de gran capacidad computacional, propios de laboratorios de grandes centros o de alta demanda de exámenes, que deben cumplir con toda la amplia gama de requerimientos planteados por la ATS (American Thoracic Society) ${ }^{4}$ o la SEPAR (Sociedad Española de Neumología y Cirugía Torácica $)^{5}$.

b) Equipos portátiles, de escritorio u oficina, de menor capacidad computacional, que deben cumplir los requisitos internacionales para las mediciones de flujo $(\mathrm{F})$, volumen $(\mathrm{V})$ y tiempo $(\mathrm{t})$ que realizan. Deben tener visualización en pantalla de las maniobras realizadas, en tiempo real e idealmente tanto de las curvas F-V y V-t, aunque bastaría la imagen de la curva de $\mathrm{F}-\mathrm{V}$, que es más clara para definir la aceptabilidad de cada maniobra.

Algunos de estos equipos pueden conectarse a un PC, mejorando la visualización de las maniobras y la impresión de los resultados.

c) Los espirómetros deben cumplir una serie de requisitos mínimos que han sido establecidos internacionalmente (Tabla 1). 
Tabla 1. Requerimientos mínimos de los espirómetros

\begin{tabular}{ll}
\hline Requerimientos & $\begin{array}{l}\text { Valores o } \\
\text { procedimientos } \\
\text { aceptables }\end{array}$ \\
\hline Margen de volumen & 0,5 a $8 \mathrm{~L}$ \\
Exactitud & $5 \%$ ó $100 \mathrm{ml}$ \\
Precisión & $3 \%$ ó $50 \mathrm{ml}$ \\
Linealidad & $3 \%$ \\
Resolución & $25-50 \mathrm{ml}$ \\
Resistencia & $<1,5 \mathrm{~cm} \mathrm{H}_{2} \mathrm{O} / \mathrm{L} / \mathrm{s}$ \\
& $($ entre $0 \mathrm{a} 14 \mathrm{~L})$ \\
Volumen mínimo detectable & $30 \mathrm{~mL}$ \\
Tiempo cero & Extrapolación retrógrada \\
Tiempo de lectura & $15 \mathrm{~s}$ \\
Señal de prueba & Jeringa de $3 \mathrm{~L}$ \\
\hline
\end{tabular}

\section{Controles de calidad de los equipos}

La periodicidad de los controles de calidad de los espirómetros depende principalmente de su tipo.

a) Control diario: Equipos grandes, de laboratorios especializados en función pulmonar.

Para todos los espirómetros:

- Calibración con una jeringa de 3 litros, mantenida a la misma temperatura y humedad que el espirómetro (requisitos de la jeringa: exactitud de $15 \mathrm{ml}$ ó $0,5 \%$ ). Debe dar un resultado dentro del 3\% del volumen de la jeringa ( $\pm 90 \mathrm{ml}$ para una jeringa de 3 litros; entre 97 y 103\%). Si la temperatura cambia más de $5{ }^{\circ} \mathrm{C}$ en el día, debe calibrarse más frecuentemente.

Para espirómetros con sensores de flujo:

- Revisión del sensor de flujo buscando perforación, obstrucción y exceso de humedad.

- Evaluación de la linealidad, que se realiza inyectando el volumen de 3 litros con 3 flujos distintos (entre 2 y 12 litros/segundo). El volumen medido para cada uno de los flujos utilizados no debe variar más de $3 \%$.

Para espirómetros basados en medición de volumen (sin sensores de flujo):

Prueba de detección de fugas. La aplicación de una presión de al menos $3 \mathrm{~cm} \mathrm{H}_{2} \mathrm{O}$ al sistema debe dar un cambio de volumen inferior a $10 \mathrm{ml} / \mathrm{min}$. b) Control trimestral:

Para espirómetros basados en medición de volumen: Evaluación de la linealidad que se realiza inyectando volúmenes de un litro seguidos de volúmenes que se incrementan en 1 litro cada vez, hasta el límite del espirómetro.

c) Controles biológicos a personal de laboratorio sano, no fumador, de distinto sexo, talla y edad (ATS recomienda al menos 3 sujetos una vez al mes):

Calcular promedio, desviación estándar (DS) y coeficiente de variación (cv).

$(\mathrm{cv}=\mathrm{DS} /$ promedio $\mathrm{x} 100)$ para cada uno de los índices espirométricos. Debe ser inferior al 10\%. Resultados fuera del promedio + 2DS requieren evaluación del equipo, la calibración y de la forma de hacer la prueba.

d) Para equipos portátiles:

- Calibración inicial y después idealmente al menos semanal, siendo lo mínimo aceptable una calibración mensual.

- Comprobar la linealidad de las mediciones haciendo inicialmente exámenes en paralelo (repetidos) con un espirómetro del laboratorio de referencia, tanto en sujetos normales como en pacientes restrictivos y obstructivos.

- Control biológico mensual.

\section{Indicaciones de la espirometría}

Las principales indicaciones de la espirometría se resumen a continuación.

\section{a) Diagnósticas:}

- Evaluación de síntomas, signos o exámenes de laboratorio relacionados con patología respiratoria.

- Valoración del impacto de enfermedades respiratorias o extra-respiratorias sobre la función pulmonar.

- Detección de individuos con riesgo de deteriorar su función pulmonar (v. gr.: fumadores).

- Evaluación de riesgo quirúrgico.

\section{b) De control}

- Control de enfermedades que afectan la función pulmonar.

- Control de pacientes expuestos a agentes nocivos para el sistema respiratorio.

- Control de reacciones adversas a drogas con toxicidad pulmonar. 
- Evaluación de la respuesta frente a intervenciones terapéuticas.

- Evaluación de pacientes con patología respiratoria en programas de rehabilitación.

\section{c) Laborales y de incapacidad:}

- Evaluación de los efectos de exposición ambiental u ocupacional.

- Evaluación del pronóstico de patologías respiratorias.

- Valoración del estado funcional respiratorio para evaluaciones laborales.

- Valoración del estado funcional respiratorio para evaluaciones de seguros.

\section{d) Epidemiológicas}

- Evaluaciones epidemiológicas.

- Derivación de ecuaciones de referencia.

- Investigación clínico-epidemiológica.

\section{Contraindicaciones}

\section{a) Relativas:}

- Falta de comprensión o colaboración con el examen.

- Dolor torácico sin causa precisada.

- Cirugía torácica reciente.

- Aneurisma aórtico no complicado.

- Aneurisma cerebral no complicado.

- Hemoptisis reciente.

b) Absolutas*:

- Síndrome coronario agudo o Infarto de miocardio menor a 1 mes.

- Neumotórax reciente (1 mes).

- Aneurisma aórtico complicado.

- Aneurisma cerebral complicado.

- Desprendimiento de retina reciente (1 mes).

- Síndrome de hipertensión endocraneana.

* Nota: En los casos de contraindicaciones absolutas es factible realizar la maniobra de capacidad vital lenta, previo acuerdo con el médico solicitante del examen.

\section{Recomendaciones al paciente previo al examen}

- Las instrucciones deben entregarse por escrito:

- No debe estar en ayunas; si se realiza en la tarde, ingerir almuerzo liviano.

- No haber realizado ejercicio vigoroso (al menos 30 minutos antes).

- No fumar al menos en la hora previa.
- Suspender el tratamiento broncodilatador, excepto que se indique lo contrario en la orden, en cuyo caso deberá quedar registrado su uso.

- Tiempo de suspensión del broncodilatador según el medicamento usado:

- $\beta-2$ adrenérgicos y anticolinérgicos por vía inhalatoria de acción corta: $\mathbf{8} \mathbf{~ h}$.

- $\beta$-2 adrenérgicos por vía inhalatoria de acción prolongada: $\mathbf{1 2} \mathbf{~ h}$

- Anticolinérgicos de acción larga: 24 h

- Teofilinas de acción prolongada: 24 h

- No suspender corticoides.

\section{Técnica}

\section{a) Consideraciones sobre higiene y control de} infecciones

Se recomienda

- Operador

- Deberá lavarse las manos antes y después de atender a cada paciente.

- Material

- Cambiar la boquilla al terminar el examen de cada paciente.

- Desinfectar, esterilizar o descartar después de cada uso: boquillas, pinzas nasales, cualquier instrumento que se ponga en contacto con la mucosa de la nariz o la boca.

- Uso de un filtro desechable en:

- pacientes con una enfermedad infecciosa transmisible

- pacientes en riesgo de adquirir infecciones por alteraciones de la inmunidad

- hemorragias pequeñas o lesiones de la mucosa bucal.

Nota: Idealmente debería usarse filtro en todos los pacientes para evitar riesgos no detectados previamente.

\section{b) Preparación del paciente}

El paciente debe estar sentado y relajado, al menos unos 5 a 10 minutos antes de la prueba. Durante este período se debe realizar una breve historia clínica, indagar sobre diagnóstico, motivo del estudio, medicación usada, enfermedades infecciosas (TBC, VIH, hepatitis), evaluando la posibilidad de contraindicaciones.

Se deberá explicar y demostrar al paciente la técnica adecuada del procedimiento que va a realizarse y cómo deberá colaborar.

Se deberá registrar:

- Nombre completo y RUT.

- Tipo y dosis de broncodilatadores usados en las últimas 8 horas. 
- Fecha de nacimiento y edad.

- Sexo.

- Peso expresado en $\mathrm{kg}$ y medido en una báscula adecuada sin zapatos y con ropa ligera.

- Estatura medida sin zapatos con la espalda erguida, la cabeza y la espalda apoyadas en la cinta de medir adherida a la pared, para mayor exactitud en la medición se puede aplicar una escuadra en la zona cefálica más alta. En sujetos con marcada cifoescoliosis u otra deformidad torácica o en pacientes que no puedan ponerse de pie, se puede estimar la talla con la medición de la envergadura (distancia máxima entre el extremo de los dedos medios de ambas manos, con las extremidades superiores extendidas al máximo en cruz).

\section{c) Ejecución del examen}

El paciente debe estar bien sentado en una silla cómoda, con brazos, con el respaldo vertical, con la espalda erguida mirando al frente y sin cruzar las piernas.

\section{d) Procedimientos}

\section{i. Capacidad vital lenta}

Se realizará en forma optativa cuando esté expresamente solicitada por el médico tratante o por el jefe del laboratorio de función pulmonar.

Esta maniobra permite medir además de la capacidad vital lenta, las subdivisiones de ésta, en especial, la capacidad inspiratoria.

- Colocación de boquilla (indeformable) en el interior de la boca, con los labios alrededor, sin interponer la lengua.

- Oclusión de la nariz con una pinza nasal.

- Activación del espirómetro por el operador.
- Respiración tranquila por la boca a volumen corriente durante no más de 5 ciclos.

- Desde el nivel de fin de espiración tranquila hasta capacidad pulmonar total: el paciente deberá realizar una inhalación rápida, pero no forzada ("debe llenarse completamente de aire").

- Después de una pausa de 1 a 2 segundos, deberá exhalar todo el aire hasta el fin del examen (se puede estimular con expresiones como "siga, siga").

- Activación de la función de término del examen por parte del operador, al alcanzarse los criterios de fin del examen.

- Desconexión del sujeto de la boquilla y retiro de la pinza nasal.

\section{ii. Capacidad vital forzada}

Esta maniobra permite medir volúmenes en el tiempo (Figura 1). Se informará:

- capacidad vital forzada (CVF),

- volumen espiratorio forzado en el primer segundo $\left(\mathrm{VEF}_{1}\right)$

- relación $\mathrm{VEF}_{1} / \mathrm{CVF}$.

La maniobra de espiración forzada tiene 3 fases:

- Inspiración máxima.

- Exhalación a máxima fuerza y velocidad.

- Exhalación continuada, completa, hasta el final del examen.

- Si se requiere una curva flujo/volumen volver a hacer una inspiración máxima forzada, ya que se imprime la curva inspiratoria realizada después de la curva espiratoria.

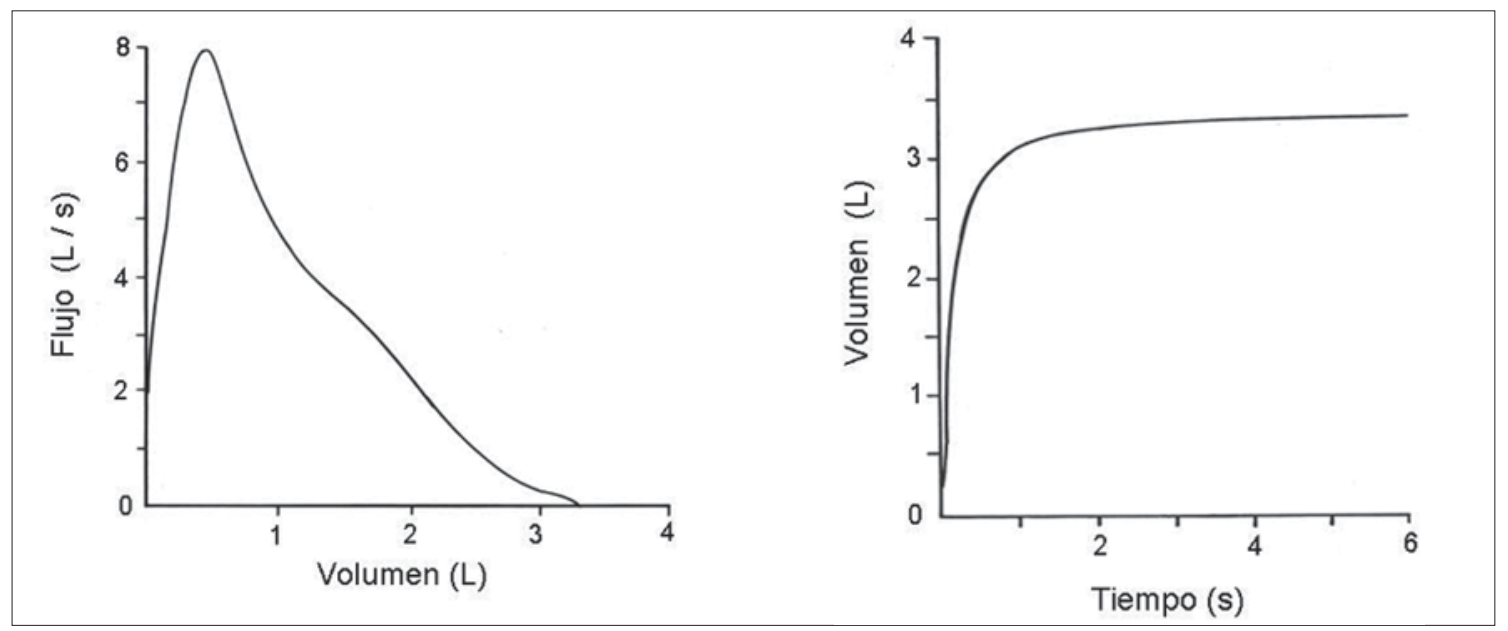

Figura 1. Espirometría aceptable. La curva de la derecha muestra una maniobra de espiración forzada en función del tiempo. La curva de la izquierda es la curva flujo-volumen de la misma maniobra. 
Procedimiento:

- Conexión del paciente a la boquilla del espirómetro.

- Oclusión de la nariz con una pinza nasal.

- Respiración a volumen corriente (no más de 5 ciclos).

- Inhalación rápida y completa desde el nivel de fin de espiración tranquila hasta capacidad pulmonar total (CPT).

- Después de una pausa menor de 1 a 2 segundos, iniciar exhalación forzada, con la máxima rapidez, por al menos 6 segundos sin detenerse, hasta alcanzar los criterios de fin de espiración.

- Nueva inhalación a la máxima velocidad llegando a CPT (sólo si se requiere analizar la curva Flujo/Volumen).

- Desconexión del sujeto de la boquilla y retiro de la pinza nasal.

\section{Criterios de fin de espiración}

- Flujos espiratorios muy bajos después de una espiración de al menos 6 segundos en adultos y niños mayores de 10 años. En niños menores de 10 años bastaría con una espiración de al menos 3 segundos.

- Haber alcanzado el "plateau" (meseta) en la curva volumen- tiempo o final asintótico en la curva flujo-volumen. Cambios menores a $0,025 \mathrm{~L}$ durante al menos 1 segundo.

- El sujeto no tolera seguir espirando.

En algunos espirómetros el fin del examen es activado por el mismo equipo al alcanzarse flujos espiratorios muy bajos $(<0,025 \mathrm{~L} / \mathrm{s}$ por al menos 1 segundo después de una espiración de al menos 6 segundos).

\section{e. Respuesta a broncodilatador}

- Utilizar salbutamol 4 inhalaciones separadas de $100 \mu \mathrm{g}$ de inhalador presurizado, a través de una cámara de inhalación de $750 \mathrm{~cm}^{3}$, siempre que no existan contraindicaciones para realizarlo.

- Si el paciente tiene antecedentes de arritmias, hipertensión arterial severa o enfermedades generadoras de temblores musculares la dosis de salbutamol será reducida a $200 \mu \mathrm{g}$.

- 15 minutos después, repetir las maniobras descritas para capacidad vital lenta (optativo) y para CVF.

- Nota. Uso del inhalador conectado a aerocámara (7) o bolsa espaciadora (8):

i) agite el inhalador y conéctelo al espaciador;

ii) ponga la boquilla del espaciador en la boca del paciente; iii) presione el inhalador una vez; iv) pida al paciente que haga una inspiración larga y lenta; v) pídale que detenga la respiración por 10 segundos y que luego exhale e inhale; vi) retire el espaciador de la boca del paciente y espere unos 30 segundos antes de realizar una segunda inhalación.

\section{Criterios de aceptabilidad y reproducibilidad}

La espirometría supondrá siempre un mínimo de tres maniobras satisfactorias de espiración forzada para conseguir los criterios de aceptabilidad y reproducibilidad y un máximo de ocho cuando no sean juzgadas adecuadas.

a) Obtener mínimo 3 maniobras aceptables de Capacidad Vital Forzada (CVF)

- Maniobras realizadas según las instrucciones previas con máximo esfuerzo.

- Los trazados no deben tener artefactos: cierre de glotis, tos, esfuerzo espiratorio variable, evidencias de fuga de aire alrededor de la boquilla (Figuras $2 \mathrm{a}, \mathrm{b}, \mathrm{c}$ y d).

- Tienen un adecuado comienzo al espirar, sin titubeos. El volumen de extrapolación retrógrada debe ser menor a $150 \mathrm{ml}$ o $5 \%$ de la CVF (Figura 2 b).

- El tiempo espiratorio $>6 \mathrm{~s}$ en adultos y niños mayores de 10 años y de $3 \mathrm{~s}$ en niños menores. Debe tratar de lograrse un plateau ( $\sin$ flujo durante $2 \mathrm{~s}$ ) como criterio de término del examen con un máximo de $15 \mathrm{~s}$ en pacientes obstruidos. No debe producirse una amputación al final de la espiración.

b) Verificar la reproducibilidad (Figuras $3 a$ y 3b) Debe haber una diferencia menor a $150 \mathrm{ml}$ entre las 2 mejores CVF y entre los 2 mejores $\mathrm{VEF}_{\mathrm{I}}$. Si la CVF es menor a 1 litro estas diferencias deben ser menores a $100 \mathrm{ml}$.

\section{Registro de Resultados}

Obtenidas al menos 3 maniobras de espiración forzada aceptables y reproducibles, con un máximo de 8 intentos:

- Registrar los valores teóricos calculados para ese sujeto.

- Elegir el mejor valor medido de CVF y $\mathrm{VEF}_{1}$ aunque sean de diferentes curvas y el $\mathrm{FEF}_{25-75}$ de la curva que tenga la suma más alta de $\mathrm{CVF}+\mathrm{VEF}_{1}$ pre-broncodilatador ("mejor curva") y registrarlo en el formulario de informe de espirometría. 


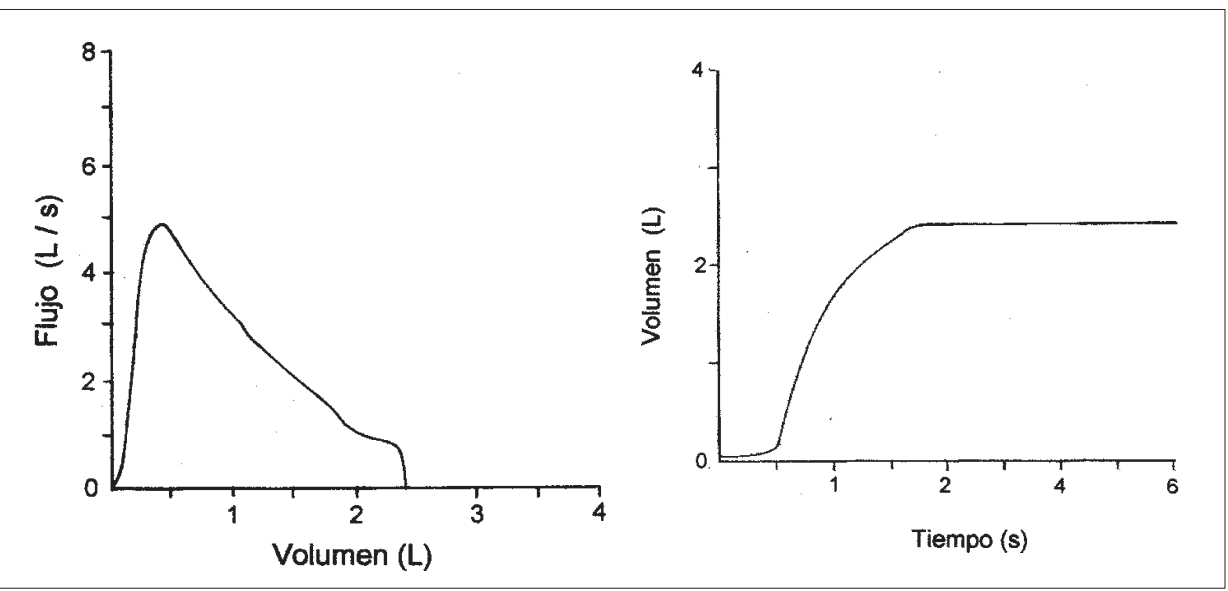

Figura 2a. Espirometría inaceptable por cierre de glotis.
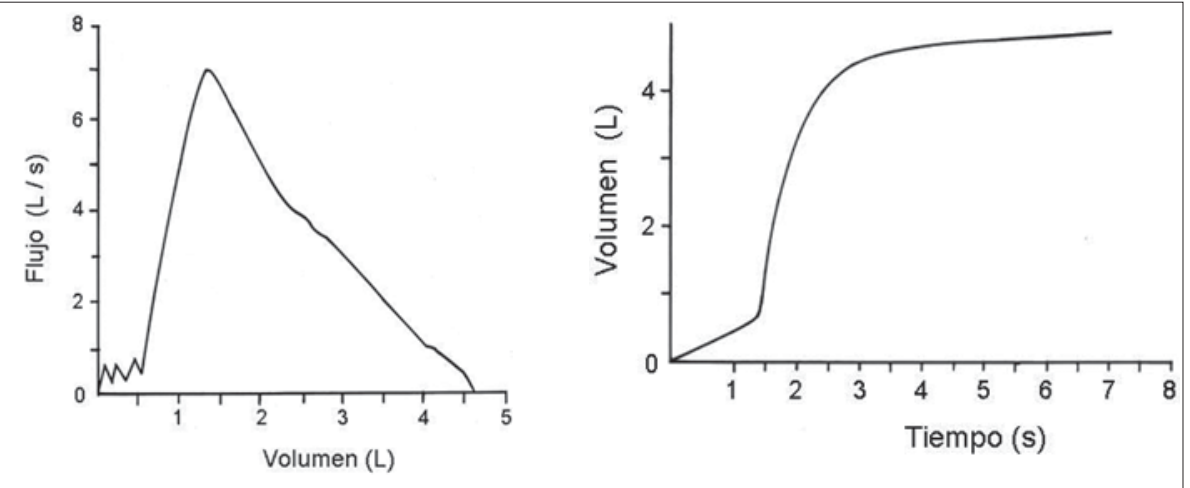

Figura 2b. Espirometría inaceptable por extrapolación de volumen $>$ $5 \%$ (extrapolación retrógada).
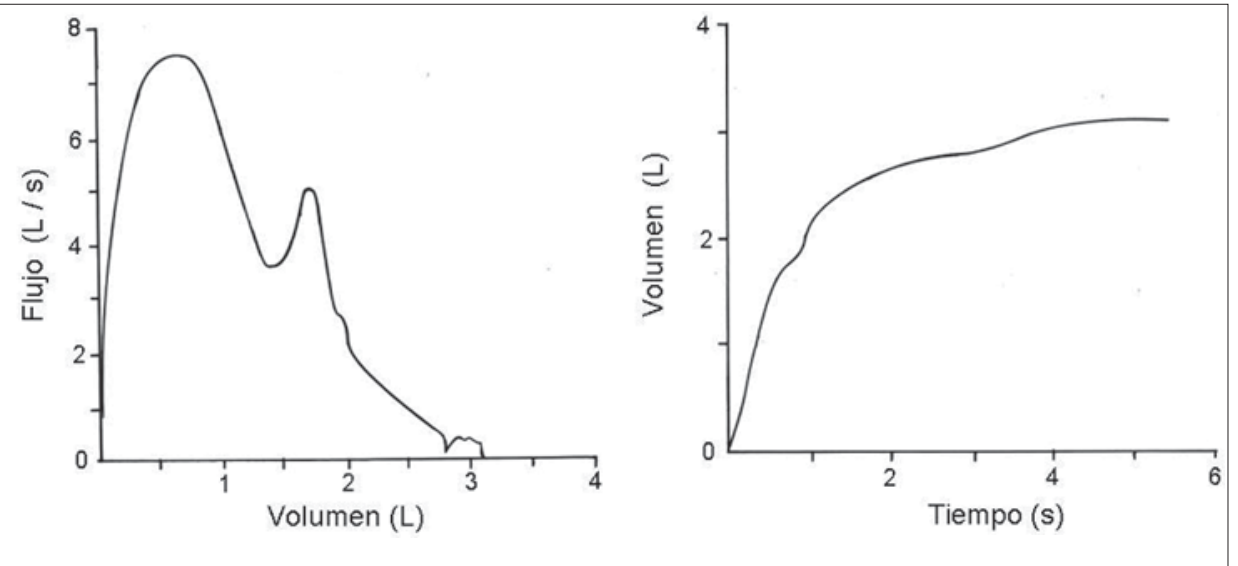

Figura 2c. Espirometría inaceptable por esfuerzo espiratorio variable comparar con Figura 1.
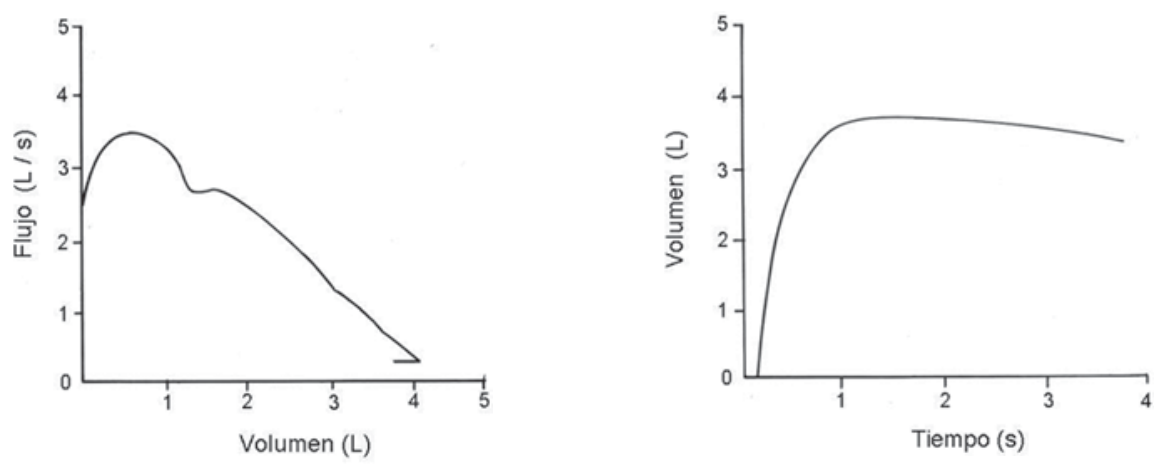

Figura 2d. Espirometría inaceptable por fuga de aire. Ver diagrama flujo-volumen (izquierda). 


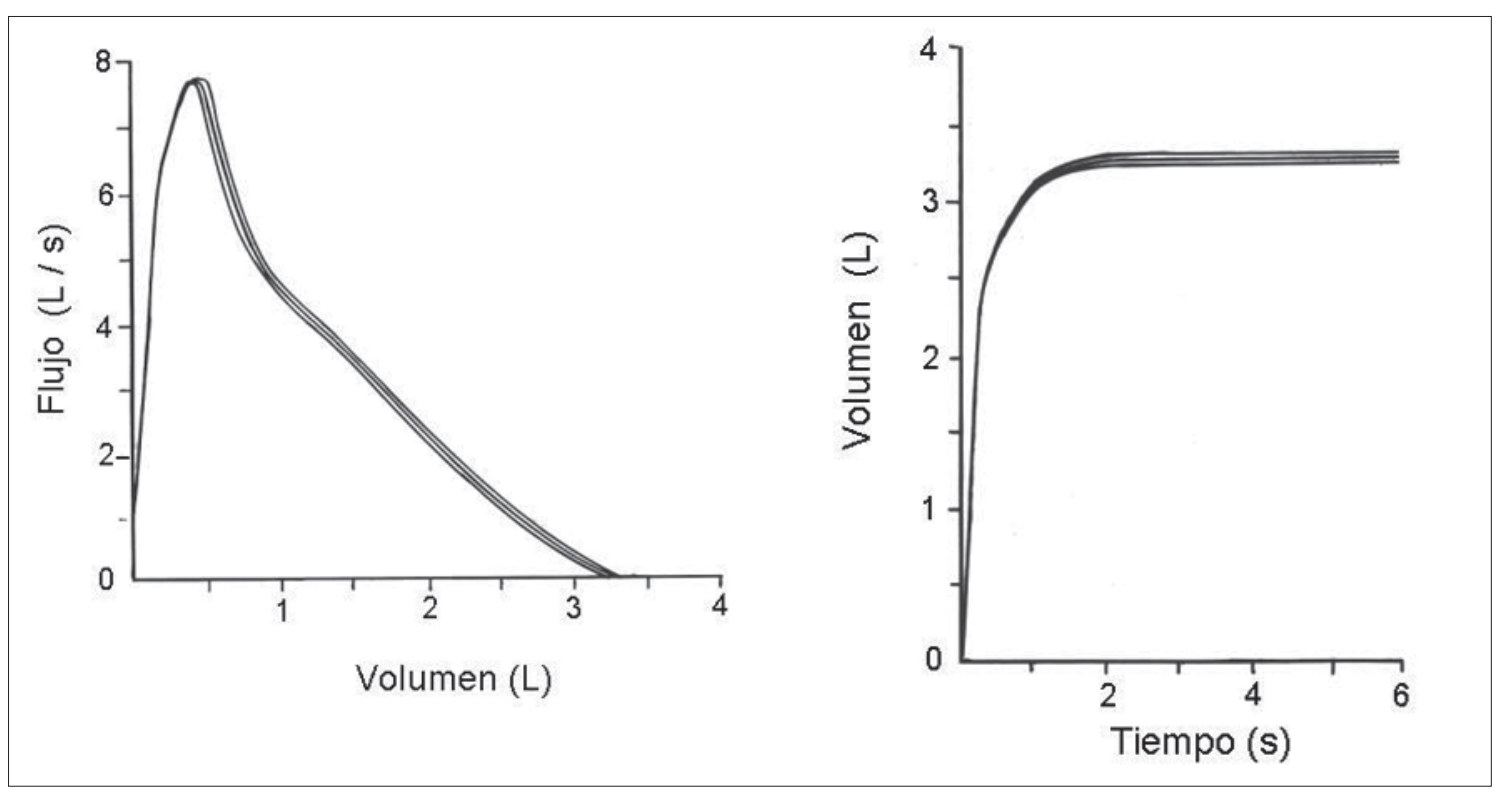

Figura 3a. Curvas espirométricas reproducibles: Se aprecia superposición de las tres curvas de espiración forzada, tanto en el diagrama flujo-volumen como en la curva volumen-tiempo.

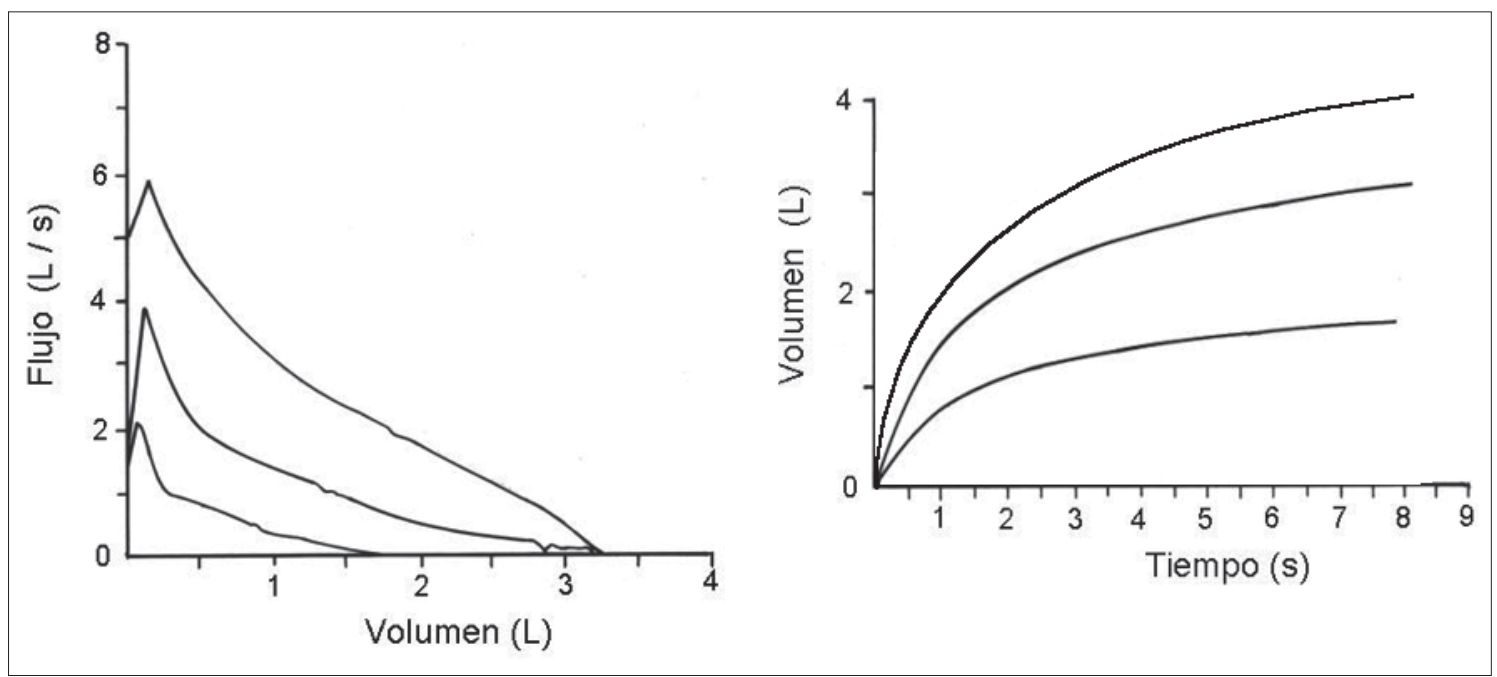

Figura 3b. Espirometría no reproducible: Se observa que las tres maniobras de espiración forzada describen diferentes diagramas flujo-volumen (izq) y volumen-tiempo (der).

- Registrar también los valores pre-broncodilatador expresados como porcentajes de los correspondientes valores teóricos.

- De la misma forma, registrar los valores de todas estas variables obtenidos después de la inhalación de broncodilatador y su cambio (diferencia en valor absoluto) expresado como porcentaje del correspondiente valor basal.

- En los equipos portátiles elegir la "mejor curva", a menos que el operador sea especialista y pueda "componer" los valores numéricos.

- En equipos grandes o portátiles siempre se imprime la mejor curva, aunque los valores hayan sido compuestos.

El registro o informe de los datos debe incluir todos los referidos a identidad y características antropométricas del paciente, fecha y lugar de realización del examen, equipo utilizado, valores de referencia y los Límites Inferiores de lo Nor- 
mal (LIN) expresados como percentil 5.

Debe incluir además las curvas flujo-volumen y volumen-tiempo para permitir evaluar la calidad de los valores obtenidos.

Los equipos grandes imprimen todas estas variables en una página que está registrada oficialmente por el fabricante, por lo cual no es necesario incluir el nombre del equipo.

Los equipos portátiles no imprimen el LIN como percentil, por lo cual es aconsejable hacer una página-informe con todas las variables ya señaladas, anotar en ella los valores numéricos y adjuntar el papel con la impresión de las curvas que entrega el equipo.

\section{$X$. Interpretación del informe de espirometría en el laboratorio}

a) Calidad del examen y comparación de los valores obtenidos con los valores de referencia

Para realizar una adecuada interpretación de los resultados obtenidos de la espirometría se debe analizar la calidad del test considerando el trazado espirométrico o gráfico volumen-tiempo y curva flujo-volumen y se deben comparar éstos con valores de referencia adecuados a la población que se está analizando. Las ecuaciones de referencia para la población de Chile, basadas en un conglomerado de mediciones realizadas en chilenos sanos de ambos sexos, con un amplio rango de edad y con una buena representación de los adultos mayores, serán publicadas próximamente, para complementar este manual.

\section{b) Patrones de alteraciones espirométricas}

El siguiente paso en la interpretación de los valores obtenidos en la espirometría, es determinar si ellos se enmarcan en alguno de los cuatro tipos de patrones espirométricos (Tabla 2) que se describen a continuación:

i) Limitación ventilatoria restrictiva: se caracteriza por una disminución de CVF y por un $\mathrm{VEF}_{\mathrm{I}}$ disminuido proporcionalmente, por lo que la relación $\mathrm{VEF}_{1} / \mathrm{CVF}$ está normal o aumentada. Una disminución concomitante del $\mathrm{FEF}_{25-75}$ no significa necesariamente que exista obstrucción bronquial, ya que la disminución de los flujos espiratorios puede ser secundaria al menor volumen pulmonar. En este caso se recomienda dejar constancia de la existencia de una disminución del $\mathrm{FEF}_{25-75}$ que debe interpretarse según el resto de la información clínica. En los casos de limitación ventilatoria restrictiva en que exista un cambio significativo de los valores con el aerosol broncodilatador, debería revisarse cuidadosamente el trazado espirométrico buscando errores técnicos; si estos no existieran, debe dejarse constancia en el informe del cambio observado.

ii) Alteración ventilatoria obstructiva con CVF normal: es aquel trastorno en que hay una disminución desproporcionada del $\mathrm{VEF}_{1}$ en relación a la $\mathrm{CVF}$, por lo que la relación $\mathrm{VEF}_{1} / \mathrm{CVF}$ está baja, es decir, menos del percentil 5 del valor predicho. La CVF obtenida antes o después del broncodilatador debe ser normal para excluir una disminución del volumen pulmonar. $\mathrm{La}$ disminución aislada de la relación $\mathrm{VEF}_{1} / \mathrm{CVF}$, con valores normales de $\mathrm{VEF}_{\text {I }}$ y $\mathrm{CVF}$, probablemente se debe a obstrucción bronquial leve en un paciente con volúmenes pulmonares grandes pero debe tenerse presente que al aumentar la edad disminuye la relación $\mathrm{VEF}_{1} / \mathrm{CVF}$ y puede constituir un falso $(+)$, aunque esto último ha disminuido al utilizar el concepto de percentil 5 como LIN.

Es necesario destacar que las alteraciones obstructivas clínicamente significativas se caracterizan por disminuciones claras del $\mathrm{VEF}_{1} \mathrm{y} / \mathrm{o}$ de la relación $\mathrm{VEF}_{1} / \mathrm{CVF}$ y que la medición del $\mathrm{FEF}_{25-75}$ no agrega información útil para calificar a un paciente como obstructivo.

iii) Limitación ventilatoria obstructiva con CVF disminuida: se caracteriza por disminución tanto de la relación $\mathrm{VEF}_{1} / \mathrm{CVF}$ como de la CVF, esto último aun después de broncodilatadores. En estos casos es conveniente asegurarse que la

Tabla 2. Características de los cuatro principales trastornos espirométricos

\begin{tabular}{lcccc}
\hline Trastorno espirométrico & $\mathbf{C V F}$ & $\mathbf{V E F}_{\mathbf{1}}$ & $\mathbf{V E F}_{\mathbf{1}} / \mathbf{C V F}$ & $\mathbf{F E F}_{\mathbf{2 5 - 7 5}}$ \\
\hline 1. Restricción & $\downarrow$ & $\downarrow$ & $\mathrm{N}$ & $\mathrm{Nó} \downarrow$ \\
2. Obstrucción con CVF normal & $\mathrm{N}$ & $\downarrow *$ & $\downarrow$ & $\downarrow$ \\
3. Obstrucción con CVF disminuida & $\downarrow$ & $\downarrow$ & $\downarrow$ & $\downarrow$ \\
4. Obstrucción mínima & $\mathrm{N}$ & $\mathrm{N}$ & $\mathrm{N}$ & $\downarrow$ \\
\hline
\end{tabular}

$\mathrm{N}=$ normal; $\downarrow=$ disminuido.

* En algunos casos el $\mathrm{VEF}_{1}$ puede ser normal (ver texto) 
espirometría haya sido técnicamente bien hecha, especialmente respecto a la duración del esfuerzo espiratorio. Sería útil medir la CV lenta que suele ser mayor que la CVF en los pacientes obstructivos.

Otra posibilidad es el colapso de la vía aérea pequeña durante la exhalación, dando como resultado un volumen residual aumentado con disminución de la CVF.

En este tipo de alteración es necesario complementar el estudio con volúmenes pulmonares, dado que la CV puede estar reducida por alteraciones restrictivas y obstructivas, la presencia de un componente restrictivo en un paciente obstructivo no puede ser inferida de una espirometría.

iv) Alteración ventilatoria obstructiva mínima: Se define como una disminución del $\mathrm{FEF}_{25-75}$ con $\mathrm{CVF}, \mathrm{VEF}_{1}$ y relación $\mathrm{VEF}_{1} / \mathrm{CVF}$ normales.

\section{c) Graduación de las alteraciones espirométricas}

Una vez establecido el tipo de alteración espirométrica, se debe determinar su intensidad. La graduación de las alteraciones es arbitraria, ya que no hay una correlación exacta entre grado de compromiso espirométrico y el deterioro de la capacidad física. Sin embargo, consideramos altamente conveniente que nuestro país cuente con criterios uniformes para informar este aspecto de la espirometría, por lo tanto recomendamos utilizar la graduación de alteraciones espirométricas resumidas en la Tabla 3. Por lo demás estos criterios son los que actualmente se usan en la gran mayoría de los laboratorios de nuestro país, lo cual demuestra su aceptación desde que fueron propuestos por la SER en $1988^{1,2}$.

Tabla 3. Graduación de las alteraciones espirométricas

\begin{tabular}{lll}
\hline $\begin{array}{l}\text { Alteración restrictiva } \\
\text { Leve }\end{array}$ & \multicolumn{1}{c}{} \\
Moderada & $\mathrm{CVF}$ & $<\mathrm{LIN} \geq 65 \%$ \\
Avanzada & $\mathrm{CVF}$ & $<50 \%$ \\
& & \\
Alteración obstructiva & & \\
Mínima & $\mathrm{FEF}_{25-75}<\mathrm{LIN}$ \\
Leve & $\mathrm{VEF}_{1} \geq 65 \%$ \\
Moderada & $\mathrm{VEF}_{1}<65 \% \geq 50 \%$ \\
Avanzada & $\mathrm{VEF}_{1}<50 \%$ \\
\hline
\end{tabular}

\section{d) Respuesta a broncodilatadores}

Para propósitos de la espirometría se interpretará como una respuesta broncodilatadora significativa a un cambio igual o mayor a $12 \%$ y $200 \mathrm{ml}$ en el $\mathrm{VEF}_{1} \mathrm{y} / \mathrm{o} \mathrm{CVF}$ respecto al valor basal, pre-broncodilatador. La estimación del efecto broncodilatador del $\mathrm{FEF}_{25-75}$ es más compleja y requiere ser calculada a iso-volumen. En estas condiciones el porcentaje de cambio de $\mathrm{FEF}_{25-75}$ debe ser superior al $30 \%$ para ser considerado significativo.

En los casos con obstrucción leve que normalizan su espirometría, sin cambios significativos del $\mathrm{VEF}_{1}$ o de la CVF se debe informar que se observó normalización de la prueba.

Se debe consignar en el informe los casos con espirometría basal normal que tienen cambios significativos de los índices espirométricos post-broncodilatador.

\section{Informe final}

Debe contener la siguiente información:

- Nombre del establecimiento donde se realizó el examen.

- Nombre del paciente.

- RUT.

- Edad, peso, talla.

- Fecha de realización.

- Tabla de resultados con valores teóricos promedio con límite inferior de normalidad y resultados obtenidos de acuerdo a instrucciones (ver item IX).

- Adjuntar gráficos con curva flujo-volumen y volumen-tiempo.

- Conclusión o interpretación.

- Indicar valores de referencia utilizados.

- Indicar el equipo en el cual se realizó el examen.

- Indicar nombre, tipo y dosis de broncodilatador utilizado.

- Nombre de la persona que realiza el examen (tecnólogo u otro).

- Nombre y firma del médico que informa el examen.

\section{Interpretación clínica de la espirometría}

El informe espirométrico que entrega el laboratorio de función pulmonar describe la interpretación funcional de los resultados. La interpretación clínica de la espirometría debe ser efectuada por el médico tratante en el contexto específico de cada paciente. 
Este manual tiene como uno de sus propósitos entregar una orientación respecto a la interpretación clínica de los resultados espirométricos en las situaciones clínicas más frecuentes.

\section{a) Enfermos con sospecha de EPOC}

De acuerdo a las normas técnicas del Programa de Control de las Enfermedades Respiratorias del Adulto en Chile, preparadas por el MINSAL, se debe sospechar EPOC en pacientes con:

- Antecedentes de tabaquismo o, menos frecuentemente, de exposición a humo de origen vegetal, como leña o contaminantes del ambiente laboral.

- Disnea, acompañada o no de tos con expectoración.

- Signos de obstrucción bronquial o de hiperinsuflación pulmonar.

Para confirmar el diagnóstico, en estos pacientes se debe demostrar la existencia de obstrucción bronquial no reversible mediante una espirometría. En estos pacientes se pueden observar los siguientes resultados de este examen:

Espirometría normal. Se descarta EPOC en etapa clínica.

Trastorno espirométrico obstructivo mínimo. Podría existir una obstrucción bronquial muy leve, como se observa en las etapas iniciales de la EPOC. En estos enfermos, los resultados espirométricos pueden ser usados para reforzar la indicación de dejar de fumar.

Trastorno espirométrico obstructivo reversible con broncodilatadores. Se descarta EPOC. $\mathrm{El}$ paciente puede tener un asma bronquial $\mathrm{u}$ otra enfermedad con obstrucción bronquial reversible.

Trastorno espirométrico obstructivo no reversible, que puede ser modificable o no modificable con aerosol broncodilatador. En estos pacientes es probable que exista una EPOC. El diagnóstico se confirma si la obstrucción no se normaliza a lo largo de algunos meses de tratamiento, confirmando el carácter no reversible de la obstrucción. Si la evolución demuestra reversibilidad a valores normales, el paciente probablemente tiene asma bronquial.

\section{b) Enfermos con sospecha de asma bronquial}

De acuerdo a las normas técnicas del Programa de Control de las Enfermedades Respiratorias del Adulto en Chile, se debe sospechar asma bronquial en pacientes con:

- Historia de asma en la infancia.

- Historia de episodios recurrentes de sibilancias, pecho apretado o disnea.

- Síntomas precipitados por ejercicio, frío, irritantes o risa.

- Alivio inmediato con broncodilatadores o alivio espontáneo en horas o días.

Para confirmar el diagnóstico de asma bronquial se debe demostrar la existencia de obstrucción reversible de las vías aéreas, definida como normalización de la función pulmonar. Esta demostración puede efectuarse mediante la espirometría o mediante el flujo espiratorio forzado máximo, más conocido como PEF (peak expiratory flow). En este manual no nos referiremos al uso de PEF, el cual puede ser consultado en una publicación previa ${ }^{9}$.

i) Espirometría normal o con trastorno espirométrico mínimo. No descarta asma bronquial, ya que el examen puede haberse efectuado entre las crisis. Si la historia es sugerente, se puede solicitar una prueba de provocación con histamina o metacolina o derivar al especialista.

ii) Trastorno obstructivo reversible con broncodilatador. Confirma el diagnóstico de asma bronquial.

iii) Trastorno espirométrico obstructivo no reversible, que puede ser modificable o no modificable con aerosol broncodilatador. El examen es compatible con asma pero no demuestra la reversibilidad, que es su sello distintivo. El diagnóstico se confirma con una espirometría normal como consecuencia del tratamiento con esteroides inhalados u orales. Se recomienda repetir la espirometría después de un tratamiento de prueba que incluye broncodilatadores y prednisona $0,5 \mathrm{mg} / \mathrm{kg}$, durante una semana. La alternativa es repetir la espirometría después de algunos meses de tratamiento con esteroides inhalados. Los enfermos con una historia sugerente de asma que no normalizan su espirometría durante su evolución, probablemente tienen un asma con remodelación de las vías aéreas.

\section{c) Etapificación de la EPOC}

De acuerdo a las normas técnicas del Programa de Control de las Enfermedades Respiratorias del Adulto en Chile, la espirometría sirve para clasificar a los pacientes con EPOC en etapa $\mathrm{A}\left(\mathrm{VEF}_{1}\right.$ mayor o igual a $50 \%$ del valor esperado) o etapa $\mathrm{B}\left(\mathrm{VEF}_{1}\right.$ inferior al $\left.50 \%\right)$. Los enfermos en etapa B deben ser derivados al especialista y en ellos se debe investigar la presencia de insuficiencia respiratoria mediante gasometría arterial. 


\section{d) Diagnóstico precoz de EPOC}

De acuerdo a las normas técnicas del Programa de Control de las Enfermedades Respiratorias del Adulto en Chile, debe solicitarse una espirometría en todo fumador mayor de 40 años, con el objetivo de detectar la enfermedad en etapa asintomática. Los criterios diagnósticos son los señalados para la sospecha de EPOC.

\section{e) Misceláneas}

Además del asma bronquial y de la EPOC, hay otras enfermedades menos frecuentes que pueden producir síntomas similares. En la mayoría de estos enfermos, el estudio diagnóstico diferencial es efectuado por el especialista. Las condiciones clínicas que pueden confundirse con asma son tos crónica (afecciones sinusales, reflujo gastroesofágico, etc), obstrucción de la vía aérea central (tumores, compresión extrínseca, etc), cuerpos extraños endobronquiales, bronquiectasias, insuficiencia cardíaca izquierda, tromboembolimo pulmonar, trastornos psiquiátricos $\mathrm{y}$ tos $\mathrm{u}$ obstrucción inducida por fármacos (bloqueadores de receptores $\beta$-adrenérgicos, inhibidores de la enzima convertidora de la angiotensina, colinérgicos).

Las condiciones que pueden confundirse con EPOC son bronquiectasias, secuelas de TBC, fibrosis quística, neumoconiosis y bronquitis obliterante.

\section{f) Otras consideraciones}

Es importante interpretar con cautela los valores cercanos a los límites inferiores de normalidad, evitando clasificarlos en alguno de los patrones espirométricos antes descritos, consignando "valores espirométricos en límites inferiores de normalidad".

Por otra parte, la ausencia de respuesta al broncodilatador durante el examen no implica una falta de respuesta clínica a la terapia broncodilatadora.

\section{Agradecimientos}

Los autores agradecen a los Drs. Edgardo Carrasco C. y Bernardita Torrealba J. por sus aportes en la discusión del documento y a las Srtas. Jeannette Quilodrán y Angélica Zamorano por el apoyo de secretaría.

\section{Bibliografía}

1.- MORENO R, OYARZÚN M. Recomendaciones sobre informe espirométrico. Primera parte. Enf Respir Cir Torác 1988; 3: 97-103.

2.- MORENO R, OYARZÚN M. Recomendaciones sobre informe espirométrico. Segunda parte. Enf Respir Cir Torác 1988; 4: 138-49.

3.- MILLER M R, CRAPO R, HANKINSON J, BRUSASCO V, BURGOS F, CASABURI R, et al. ATS/ERS task force: Standardisation of lung function testing': General Considerations for lung function testing. Eur Respir J 2005; 26: 153-61.

4.- MILLER M R, HANKINSON J, BRUSASCO V, BURGOS F, CASABURI R, COATES A, et al. Series 'ATS/ERS task force: Standardisation of lung function testing': Standardisation of spirometry. Eur Respir J 2005; 26: 319-38.

5.- CASAN P, BURGOS F, BARBERÀ J A, GINER J. Espirometría Manual SEPAR de Procedimientos. Módulo 3. Procedimientos de evaluación de la función pulmonar: 2002. SEPAR. ISBN Obra completa: 847989-152-1 ISBN Módulo 3: 84-7989-155-6.

6.- GIBSON G J. Spirometry: then and now. Breathe 2005; 1: 207-16.

7.- PINO J, CERECEDA J. Educación sobre asma en adultos. Rev Chil Enf Respir 1995: 193a -197a.

8.- MENDOZA L, CONTRERAS C, ESPINOZA A, JOVER E, CAVADA G, et al. El sistema de la bolsa es útil como espaciador para la administración de los aerosoles presurizados. Rev Chil Enf Respir 2005; 21 : 33-8.

9.- SEPÚLVEDA R. El flujómetro de Wright: Una herramienta indispensable en la práctica ambulatoria. Rev Chil Enf Respir 2004; 20: 80-4.

Correspondencia a:

Dra. Mónica Gutiérrez Clavería

E-mail: mgutierrezc@hospitalnaval.cl mgancaroe@gmail.com 